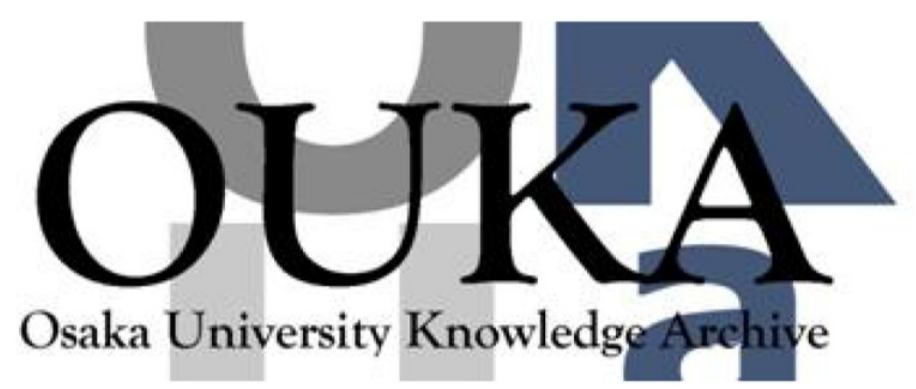

\begin{tabular}{|c|c|}
\hline Title & Proton Radiography of a Laser-Driven Implosion \\
\hline Author (s) & $\begin{array}{l}\text { Mackinnon, A. J.; Patel, P. K.; Borghesi, M. et } \\
\text { al. }\end{array}$ \\
\hline Citation & Physical Review Letters. 97(4) p. 045001 \\
\hline Issue Date & $2006-08$ \\
\hline oaire:version & VoR \\
\hline URL & https://hdl. handle. net/11094/3384 \\
\hline rights & $\begin{array}{l}\text { Mackinnon, A. J., Patel, P. K., Borghesi, M., } \\
\text { Clarke, R. C., Freeman, R. R., Habara, H., } \\
\text { Hatchett, S. P.' Hey, D., Hicks, D. G., Kar, } \\
\text { S., Key, M. H.'King, J.' A., Lancaster, K., } \\
\text { Neely, D.' Nikkro, A.,' Norreys, P. A., Notley, } \\
\text { M. M., Phillips, T. W., Romagnani, L.,' Snavely, } \\
\text { R. A.', Stephens, R. B.,' Town, R. P. J., } \\
\text { Physical Review Letters, 97, 4, 045001, 2006- } \\
\text { 08. "Copyright } 2006 \text { by the American Physical } \\
\text { Society." }\end{array}$ \\
\hline Note & \\
\hline
\end{tabular}

Osaka University Knowledge Archive : OUKA

https://ir. Library. osaka-u. ac. jp/ 


\title{
Proton Radiography of a Laser-Driven Implosion
}

\author{
A. J. Mackinnon, ${ }^{1}$ P. K. Patel, ${ }^{1}$ M. Borghesi, ${ }^{2}$ R. C. Clarke,${ }^{4}$ R. R. Freeman, ${ }^{4}$ H. Habara, ${ }^{4}$ S. P. Hatchett, ${ }^{1}$ D. Hey, ${ }^{1}$ \\ D. G. Hicks, ${ }^{1}$ S. Kar, ${ }^{2}$ M. H. Key, ${ }^{1}$ J. A. King, ${ }^{3}$ K. Lancaster, ${ }^{4}$ D. Neely, ${ }^{4}$ A. Nikkro, ${ }^{5}$ P. A. Norreys, ${ }^{4}$ M. M. Notley, ${ }^{4}$ \\ T. W. Phillips, ${ }^{1}$ L. Romagnani, ${ }^{2}$ R. A. Snavely, ${ }^{1}$ R. B. Stephens,${ }^{5}$ and R. P. J. Town ${ }^{1}$ \\ ${ }^{1}$ Lawrence Livermore National Laboratory, Livermore, California 94550, USA \\ ${ }^{2}$ Queen University, University Road, Belfast BT7 1NN, Northern Ireland, United Kingdom \\ ${ }^{3}$ Department of Applied Science, University of California, Davis, California 95616, USA \\ ${ }^{4}$ Rutherford Appleton Laboratory, Chilton, Oxon OX11 OQX, United Kingdom \\ ${ }^{5}$ General Atomics, 3550 General Atomics Court, San Diego, California 92121, USA
}

(Received 25 March 2005; published 24 July 2006)

\begin{abstract}
Protons accelerated by a picosecond laser pulse have been used to radiograph a $500 \mu \mathrm{m}$ diameter capsule, imploded with $300 \mathrm{~J}$ of laser light in 6 symmetrically incident beams of wavelength $1.054 \mu \mathrm{m}$ and pulse length $1 \mathrm{~ns}$. Point projection proton backlighting was used to characterize the density gradients at discrete times through the implosion. Asymmetries were diagnosed both during the early and stagnation stages of the implosion. Comparison with analytic scattering theory and simple Monte Carlo simulations were consistent with a $3 \pm 1 \mathrm{~g} / \mathrm{cm}^{3}$ core with diameter $85 \pm 10 \mu \mathrm{m}$. Scaling simulations show that protons $>50 \mathrm{MeV}$ are required to diagnose asymmetry in ignition scale conditions.
\end{abstract}

DOI: 10.1103/PhysRevLett.97.045001

The conditions leading to ignition of inertial confinement fusion (ICF) capsules are extremely difficult to realize. In conventional ICF, cryogenic deuterium tritium fuel is compressed from an initial diameter of $2 \mathrm{~mm}$ to a $60 \mu \mathrm{m}$ diameter hot spot, resulting in particle densities $(\rho)$ ranging from $300-600 \mathrm{~g} / \mathrm{cm}^{3}$ around the cold fuel-hot spot interface and an areal density $(\rho R)$ of $0.3 \mathrm{~g} \mathrm{~cm}^{-2}$ inside the hot spot. To achieve ignition the compressed core must be spherically symmetric, with a relatively smooth interface between the cold fuel and the hot spot [1]. Diagnosing density perturbations and general deformity of the core shape in these conditions is a challenge to conventional radiographic techniques. The ideal diagnostic would penetrate densities up to $600 \mathrm{~g} \mathrm{~cm}^{3}$ and resolve features of less than 5-10 $\mu \mathrm{m}$. Imaging of neutron emission from an igniting core is capable of high resolution imaging during ignition shots [2], however it is unclear whether a target that does not reach ignition will produce enough neutrons to form a good image.

Advanced diagnostic techniques that can characterize dynamically evolving conditions in highly compressed media are clearly desirable. One possible method, currently under development is x-ray imaging in the range of $15-30 \mathrm{keV}$ [3]. An alternative, perhaps complementary, technique utilizes laser-driven proton beams of 30$100 \mathrm{MeV}$ to probe density perturbations in highly dense materials. Multiple scattering arising from density structures in the probed object modulates the proton beam, thus allowing micron scale structures to be imaged onto the film plane $[4,5]$. Proton probing experiments of electromagnetic fields in low-density laser-produced plasmas have been carried out [4], however, the utility of proton radiography in dense laser-compressed materials created by laser-driven implosion has yet to be studied. Protons beams with substantial particle fluxes at $40-50 \mathrm{MeV}$ have been
PACS numbers: 52.50.Jm, 52.40.Nk, 52.40.Mj, 52.70.Kz

produced by focusing a petawatt (PW) laser pulse on a thin foil target [6]. These protons can penetrate $\rho=$ $500-1000 \mathrm{~g} \mathrm{~cm}^{3}$ and so picosecond laser-driven proton radiography is a potential candidate as a probe of dynamic events in extremely dense matter.

This Letter reports on the first proof of principle study of dynamic proton probing to diagnose density gradients in a laser-driven spherical implosion. The experiment was carried out using the $100 \mathrm{TW}, 1$ ps Vulcan laser pulse coupled to a six-beam laser-driven implosion system [7]. The compressed core was characterized by protons and verified using x-ray radiography; the experiments were simulated by the 1D hydrocode (HYDRA [8]) and a 3D Monte Carlo (MC) particle tracing code based on SRIM [9].

The protons were produced from a $1.054 \mu \mathrm{m}$ wavelength laser with 1 ps pulse duration and $50 \mathrm{~J}$ energy, focused by an $F / 3.5$ off-axis parabola close to normal incidence onto a $25 \mu \mathrm{m}$ thick tungsten target. The focal spot (containing 30\%-40\% of the energy) was $10 \mu \mathrm{m}$ full-width at half maximum (FWHM), giving a peak irradiance of $5 \times 10^{19} \mathrm{~W} / \mathrm{cm}^{2}$. This interaction produced a proton beam with an exponential spectrum of mean energy $1.5 \mathrm{MeV}$ and a high-energy cutoff around $10-15 \mathrm{MeV}$. The proton producing target was protected from the plasma surrounding the imploded target with a $6 \mu \mathrm{m}$ thick $\mathrm{Al}$ filter, an arrangement that preserved the fidelity of the proton beam while maintaining good spatial resolution in the proton images [10].

The implosion was driven by six laser beams, each $1 \mu \mathrm{m}$ wavelength and $1 \mathrm{~ns}$ duration, focused onto a microballoon at an irradiance of $1 \times 10^{13} \mathrm{Wcm}^{-2}$. These beams were derived from the same laser oscillator as the short pulse beam, thus eliminating any relative timing jitter between implosion beams and the proton backlighter. The targets were plastic (poly- $\alpha$-methyl styrene) microbal- 
loons, $500 \mu \mathrm{m}$ in diameter and with wall thickness either 3 or $7 \mu \mathrm{m}$ and no fill. Individual beam energy was in the range of $50 \mathrm{~J}$, giving a maximum energy on target of $300 \mathrm{~J}$ and the beams were arranged such that they illuminated the target tangentially from 6 orthogonal directions, giving the best symmetry for a six-beam implosion, as shown in Fig. 1. The synchronization of the $1 \mathrm{~ns}$ heater beams to the picosecond backlighter beam was measured to within accuracy of $100 \mathrm{ps}$ using an optical streak camera.

The proton detector used in this experiment consisted of a multilayer film pack containing spatially resolving radiochromic (RCF) dosimetry film (types: MD-55 and HD810). This arrangement, which has been extensively used in laser-driven proton acceleration experiments, gave a diagnostic in which each layer was filtered by the preceding layer, giving a series of images per shot, each with a slightly different energy, ranging from 3 to $15 \mathrm{MeV}$. The propagation of protons through the radiography object was modeled using a MC simulation code based on SRIM [8]. SRIM calculates the final spatial and energy distribution of ions passing through the object, taking into account ionization energy loss by the proton into the target and energy transferred to recoil atoms. The simulation code uses SRIM to record the energy and position of each proton as it passes through the target and the specific RCF film pack used in the experiment. The program calculates the dose deposited in each RCF layer by adding up the contributions from each incident proton. This procedure provides a radiographic projection of a transverse $2 \mathrm{D}$ slice of the target. If the object has spherical symmetry then a threedimensional object can be obtained by superimposing a large number of profiles rotated in small angles about the center [11].

A static test of point projection proton imaging was obtained by backlighting an undriven microballoon with diameter $500 \mu \mathrm{m}$ and wall thickness $7 \mu \mathrm{m}$. The image of the balloon in $7 \pm 1 \mathrm{MeV}$ protons shown in Fig. 2(a) was obtained by projecting the proton beam onto the film pack with a magnification of $10 \times$ (in this case the 3 rd layer of the film pack). A profile through the center of the shell is shown in Fig. 2(b). It is clear that the balloon strongly

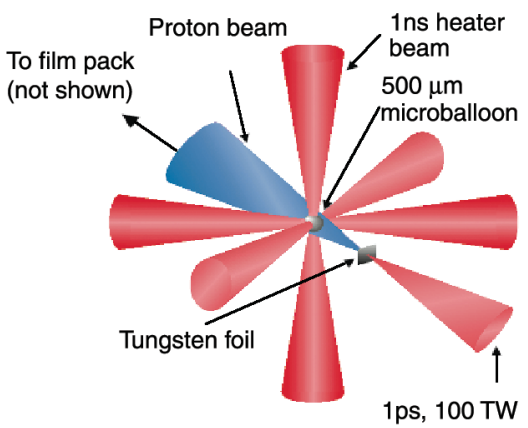

laser beam

FIG. 1 (color). Geometry for proton radiography of the 6 beam implosion. modulates the proton beam, with the highest modulation occurring at the edges of the shell where the path length through solid density material is highest. Also shown on Fig. 2(b) is the output of 3D proton Monte-Carlo simulations of $7 \mathrm{MeV}$ protons from a point source propagating through the microballoon. The simulations agree well with the data, reproducing the shape of the edge of the balloon and confirming that multiple small angle scattering accounts for the image formation.

Temporal evolution of the capsule density profile during the implosion was studied by varying the delay between the backlighter and the implosion beams. The temporal resolution of these proton radiographs is determined by the energy bandwidth of each detector layer and the time of flight differences for these protons as they propagate from the source foil to the target plane. This results in two effects: (1) each film layer probes a slightly different time in the implosion and (2) time smearing within each layer of film is determined by the energy bandwidth of the film. The time smearing effect can be calculated knowing the center energy and film layer bandwidth. For instance for the third layer of film, with center energy of $7 \mathrm{MeV}$, $1 \mathrm{MeV}$ bandwidth, and a source to object distance of $4 \mathrm{~mm}$, the temporal smearing is $\sim 10 \mathrm{ps}$. This film layer is easily able to freeze motion in inertial confinement fusion (ICF) plasmas where time scales for motion are typically $>50-100 \mathrm{ps}$. The time difference between the arrival of 5 and $10 \mathrm{MeV}$ protons at the target is $30 \mathrm{ps,}$, so no additional information can be obtained by comparing different film layers in this relatively slowly evolving plasma, however this feature has been used in other experiments to investigate sheath field evolution on ps time scales [4].

With nominally symmetrical drive conditions the capsule remains roughly spherical as the implosion proceeds, as can be seen from Fig. 3(a). This $7 \mathrm{MeV}$ proton radiograph, taken $2 \mathrm{~ns}$ after the start (defined as the time where the heater beam intensity reaches half maximum) of the implosion, shows that the capsule has retained roughly spherical symmetry with the shell diameter reducing from 500 to $300 \mu \mathrm{m}$ and capsule walls that are still largely
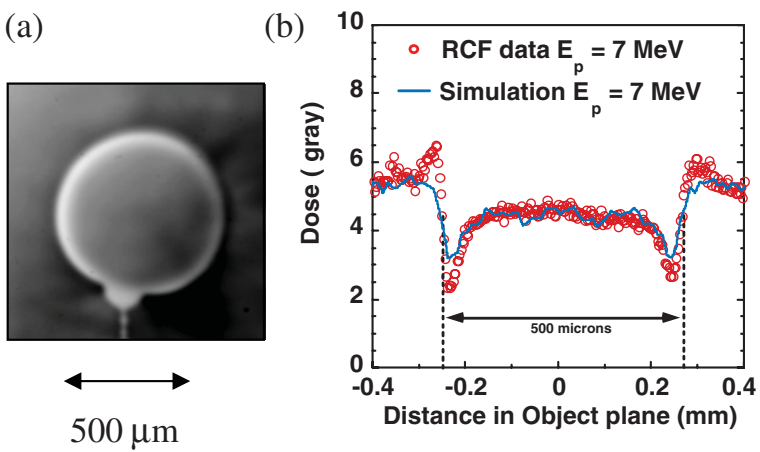

FIG. 2 (color). (a) $7 \mathrm{MeV}$ Proton radiograph of undriven $500 \mu \mathrm{m}$ diameter, 7 um wall thickness shell. (b) Lineout through data (red circles) and Monte Carlo simulation (blue line) of proton propagation through shell and RCF film pack. 

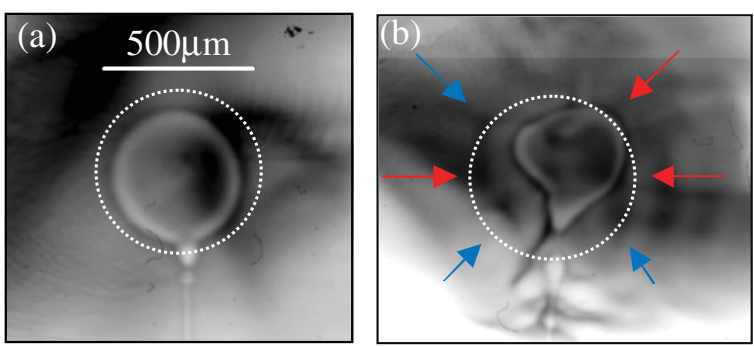

FIG. 3 (color). (a) Proton radiograph (7 MeV protons) of quasisymmetric implosion, taken $2 \mathrm{~ns}$ after the peak of the drive beams. (b) Proton radiograph taken of highly asymmetric implosion, caused by mistimed heater beams. Beams colored in blue arrived 1-2 ns earlier than beams colored in red.

intact. There is some residual asymmetry due to the imperfect synchronization and energy balance of the 6 heater beams; the shell is slightly elliptical, having departed from its original spherical shape. These features are consistent with 300-500 ps synchronization errors that were present in the relative timing of the heater beams. In contrast Fig. 3(b) shows a proton image of a capsule where the drive was very asymmetrical due to significant timing difference between the drive beams. In this case there were delays of up to $2 \mathrm{~ns}$ between the heater beams, leading to large distortions from spherical symmetry.

Radiographs were also taken closer to the time of peak density (stagnation) of the implosion of a $3 \mu \mathrm{m}$ wall thickness shell. The $5 \mathrm{MeV}$ proton radiograph (the highest proton energy image available for this shot) in Fig. 4(a) was taken at $3 \mathrm{~ns}$, which is close to stagnation for this shell. At this time a dense core has assembled just below the center point of the original capsule, due to the higher drive levels from the upper beams. This results in a core that is slightly elliptical with a minor diameter of $120 \mu \mathrm{m}$ and a major diameter of $140 \mu \mathrm{m}$ (FWHM). The radiograph

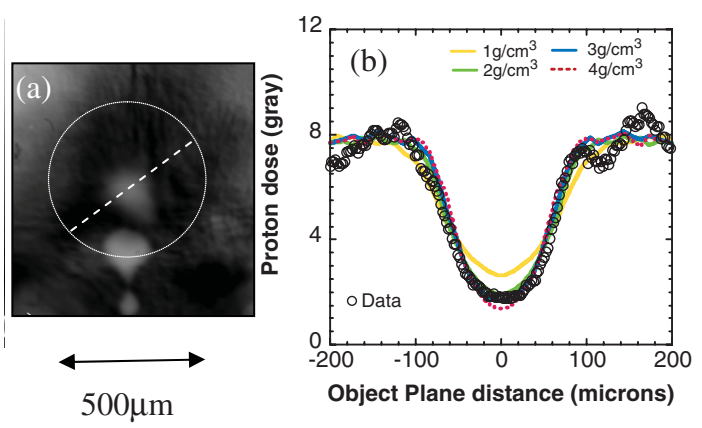

FIG. 4 (color). (a) Proton radiograph, in $7 \mathrm{MeV}$ protons, of a $500 \mu \mathrm{m}$ diameter microballoon with a $3 \mu \mathrm{m}$ wall at a time close to stagnation (To $+3 \mathrm{~ns}$ ). (b) Radial lineout taken through center of proton radiograph data (blue circles) through the minor axis and Monte Carlo simulation output for variable peak density and varying core size (FWHM) while keeping the total mass constant. The blue curve shows the best fit of $3 \mathrm{~g} / \mathrm{cm}^{3}$ peak and FWHM of $83 \mu \mathrm{m}$. Yellow: $1 \mathrm{~g} / \mathrm{cm}^{3}, 120 \mu \mathrm{m}$ FWHM; Green: $2 \mathrm{~g} / \mathrm{cm}^{3}, 95 \mu \mathrm{m}$ FWHM; dashed red: $4 \mathrm{~g} / \mathrm{cm}^{3}, 75 \mu \mathrm{m}$ FWHM. clearly resolves this asymmetry (it is important to note that the primary goal of this experiment was not to achieve a perfect implosion but to prove the capability and effectiveness of protons as a radiography source to diagnose departures from symmetry).

In order to quantify the core density, the $\mathrm{MC}$ simulation code was used to model the propagation of a protons with a $1.5 \mathrm{MeV}$ slope temperature through a spherically symmetric object assuming a Gaussian radial profile and then onto the film plane with a magnification of 10 . The simulation accounted for scattering in the dense core and the film pack to allow a direct comparison with the data. The peak density and FWHM of the Gaussian profile were treated as variable parameters until the best fit with the observed radiographs was obtained, with the background level of the simulations scaled to match the data background. Figure 4(b) shows a profile across the minor diameter of the radiograph of the core, together with the output radiograph from Monte Carlo simulations. The Gaussian source function was varied from $1-4 \mathrm{~g} \mathrm{~cm}^{3}$ while the source diameter (FWHM) was varied from 120 to $75 \mu \mathrm{m}$, respectively, (thus keeping the total mass constant) until the simulated radiograph matched the measured one. The best fit to the data for the source density was $3 \pm$ $1 \mathrm{~g} \mathrm{~cm}^{3}$ with a core diameter (FWHM) equal to $85 \pm$ $10 \mu \mathrm{m}$ with the assumption of a Gaussian density profile. These results agreed well (within 25\%) to the density and core size measured using $K_{\alpha}$ radiography of this implosion [12].

Simple analytical estimates of multiple scattering from Highland's formula, were used as an approximate check on the detailed MC simulations [13]. Particles of momentum $p$ (in units of $\mathrm{MeV}$ ), velocity, $v$ and charge $z$, traversing a material length $L$ (in units of $\mathrm{g} \mathrm{cm}^{-2}$ ) are scattered with rms angle $\Theta_{1 / e}[\mathrm{rad}]=z(E s / p \beta c)\left(L / L_{\mathrm{rad}}\right)^{1 / 2}$, where $E s=$ constant $=15, L_{\mathrm{rad}}$ is the radiation length, a $Z$ dependent constant for each material [14], and $\beta=v / c$. For $5 \mathrm{MeV}$ protons traversing $80 \mu \mathrm{m}$ of carbon at $3 \mathrm{~g} / \mathrm{cm}^{3}$, $z=1, \quad p \beta c=10 \mathrm{MeV}, \quad L \sim 80 \mu \mathrm{m} \times 3 \mathrm{~g} / \mathrm{cm}^{3}=$ $0.024 \mathrm{~g} \mathrm{~cm}^{-2}, \quad L_{\text {rad }}=43.4 \mathrm{~g} \mathrm{~cm}^{-2}$ for carbon, giving $\Theta_{1 / e}[\mathrm{rad}]=0.035 \sim 2^{\circ}$. For an object to film plane distance, $d=40 \mathrm{~mm}$, a scattering angle of $2^{\circ}$ would lead to a blur of $d^{*} \Theta_{1 / e}=1400 \mu \mathrm{m}$. Dividing by the magnification $(M=10)$ gives $140 \mu \mathrm{m}$ at the object plane. The approximate size of the image at the object plane would then be convolution of the initial source size and the blurring $\sim\left(80^{2}+140^{2}\right)^{1 / 2}=160 \mu \mathrm{m}$. This simple calculation agrees well (to within 30\%) to the core size of $120 \mu \mathrm{m}$ observed in the radiographs. In general, blurring resolution can be improved by using more energetic protons to minimize multiple scattering. For example, $15 \mathrm{Mev}$ protons gives $\Theta_{1 / e} \sim 0.6^{\circ}$ for these object conditions, reducing the blurring from 140 to $35 \mu \mathrm{m}$.

The feasibility of proton radiography for diagnosing density uniformity in very dense objects such as a compressed ICF shell was investigated by using the COG MC 


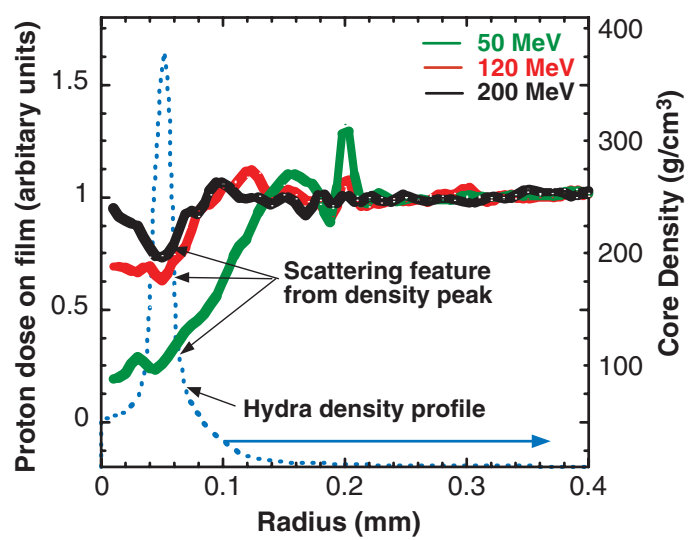

FIG. 5 (color). Simulated dose profiles taken with 50, 120, and $200 \mathrm{MeV}$ protons propagating through a density profile of a mistimed National Ignition Facility implosion obtained from 1D Hydra simulation (blue dashed line).

model [15] to simulate radiographs of a density profile generated by a 1D HYDRA simulation of a subignition national ignition facility implosion. Figure 5 shows a HYDRA profile of a "failed" indirect drive national ignition facility implosion, where some of the shocks used to assemble the high-density fuel have been mistimed, together with simulated proton radiographs at three energies. Density conditions inside this nonigniting core are still extremely high, with a peak core density up to $350 \mathrm{~g} / \mathrm{cm}^{3}$. The profile shows a high-density shell surrounding a hollow core at stagnation. In this case the peak core density of $350 \mathrm{~g} / \mathrm{cm}^{3}$ is located at a radius of $50 \mu \mathrm{m}$, surrounding a central region with density $50 \mathrm{~g} / \mathrm{cm}^{3}$. Simulations of a point projection proton image of the object using monochromatic 50,120 , or $200 \mathrm{MeV}$ protons are shown in Fig. 5. In these simulations the film was filtered with $6 \mathrm{~mm}$ of gold to slow down the highenergy protons before encountering the first RCF layer (multiple scattering in the gold filter is significant for the $50 \mathrm{MeV}$ protons, but it is mitigated by the closeness of the scattering material to the detection plane [16]). It can be seen that the density peak at $50 \mu \mathrm{m}$ radius is clearly resolved using 120 or $200 \mathrm{MeV}$ protons but only marginally with $50 \mathrm{Mev}$ protons. Proton beams with $50 \mathrm{MeV}$ have already been produced by PW laser systems [6], however, the generation of sufficient $>120 \mathrm{MeV}$ protons would require a significant improvement in the high-energy cutoff of laser-driven proton beams.

Macroscopic electric fields driven by pressure gradients in the compressed core will also deflect protons. For an ignition scale implosion the electric fields are driven by pressure gradients in the core of order of the ignition temperature $T$, over the core size $r ; T / r \sim 10 \mathrm{keV} / 30 \mu \mathrm{m} \sim$ $3 \times 10^{6} \mathrm{~V} \mathrm{~cm}^{-1}$. The deflection angle can be estimated from the change in transverse momentum of the protons induced by the field as it passes through the core. For a constant field acting over a distance, $x$, the deflection angle $\theta_{[\mathrm{rad}]}=e E x /\left(2 E_{\mathrm{kin}}\right)$, where $e$ is the proton charge, $E$ is the electric field, $x$ is the core radius, and $E_{\text {kin }}$ is the particles kinetic energy. For $E \sim 3 \times 10^{6} \mathrm{~V} \mathrm{~cm}^{-1}, x=30 \mu \mathrm{m}$ and $50 \mathrm{MeV}$ protons, the deflection angle, $\theta<0.01^{\circ}$. This would not be detectable compared to multiple scattering $\Theta_{1 / e} \sim 1^{\circ}$ for these conditions. From these estimates it is clear that in the high-density core of a stagnating implosion, typical of laser-driven ICF, multiple scattering would always tend to dominate over deflections from pressure gradient driven electric fields.

In conclusion proton radiography of a laser-driven implosion with $10 \mathrm{ps}$ time resolution has been demonstrated. MC simulations through a core with a Gaussian density profile agreed well with the experimental data. MC simulations also showed that protons $>50 \mathrm{MeV}$ would be required to resolve core symmetry in highly dense plasmas such as those predicted at ignition scale.

This work was performed under the auspices of the U.S. Department of Energy by the Lawrence Livermore National Laboratory under Contract No. W-7405-ENF-48 and EPSRC Grants. A.J.M. acknowledges discussions with O. Landen and T. E. Cowan and partial support from the I.R.C.E.P., Queens University, Belfast.

[1] J. Lindl et al., Phys. Plasmas 11, 339 (2004).

[2] M. J. Moran et al., Rev. Sci. Instrum. 74, 1701 (2003); 75, 3592 (2004).

[3] J. Koch et al., Rev. Sci. Instrum. 74, 2130 (2003).

[4] M. Borghesi, et al., Plasma Phys. Controlled Fusion 43, A267 (2001); , Phys. Plasmas 9, 2214 (2002).

[5] J. Cobble et al., J. Appl. Phys. 921775 (2002).

[6] R. Snavely et al., Phys. Rev. Lett. 85, 2945 (2000).

[7] C. N. Danson et al., J. Mod. Opt. 45, 1653 (1998).

[8] M. Marinak, Phys. Plasmas 5, 1125 (1998).

[9] http://www.SRIM.org; J.F. Zeigler, The Stopping and Range of Ions in Matter (Pergamon, New York, 1985), Vol. 1.

[10] A. J. Mackinnon et al., Phys. Rev. Lett.. 86, 1769 (2001); M. Roth, Phys. Rev. ST Accel. Beams 5061301 (2002).

[11] L. Romagnani et al., RAL Annual report 01/02, 2002, 26; S. Kar et al., RAL Annual report 03/04, 2004, 26.

[12] J. King et al., Appl. Phys. Lett. 86, 191501 (2005).

[13] V. L. Highland, Nucl. Instrum. Methods 129, 497 (1975).

[14] T. A. Lasinski et al., Particle Data Group, Rev. Mod. Phys. 45, S1 (1973).

[15] COG can be obtained from ETSC, P.O. Box 1020 Oak Ridge, TN 37831-1020, USA.

[16] Multiple scattering of $50 \mathrm{MeV}$ protons in $6 \mathrm{~mm}$ gold gives a resolution element of $\sim 0.8 \mathrm{~mm}$ at the film plane, resulting in spatial resolution of $\sim 20 \mu \mathrm{m}$ for $40 \times$ magnification. 